International Mathematical Forum, Vol. 8, 2013, no. 37, 1839 - 1851 HIKARI Ltd, www.m-hikari.com http://dx.doi.org/10.12988/imf.2013.310185

\title{
On the Use of Strategies Attached to Subpopulations in the Description of Competition among Different Populations
}

\author{
Thomas Wieder \\ https://sites.google.com/site/twieder21stcenturyad \\ Copyright (c) 2013 Thomas Wieder. This is an open access article distributed under the \\ Creative Commons Attribution License, which permits unrestricted use, distribution, and \\ reproduction in any medium, provided the original work is properly cited.
}

\begin{abstract}
We propose a most simple model for the growth rates of interacting populations. Each population is composed of subpopulations and each subpopulation interacts with each other subpopulation according to a certain strategy. The interactions are represented as products of the subpopluation numbers attached with coefficients which represent certain strategies. The observable effect of a strategy is just the resulting growth rate. Identification and activity are the strategies considered here and subpopulations are either susceptible or insusceptible to these strategies. By utilizing identification and activity each population competes for growth at the cost of the other populations. An ordinary differential equation system follows as a simple quantitative model for the population growth. No actual forecast is calculated but we critically discuss the adequateness of the model compared to the sociological reality.
\end{abstract}

Mathematics Subject Classification: 91D10

Keywords: sociophysics, population dynamics, social strategies

\section{Introduction}

Sociophysics is a branch of physics which applies physical methods on social systems in order to describe sociological phenomena [1, 2]. Among the 
subjects of sociophysics we find opinion dynamics [3], ideological struggle [4], the dynamics of party formation [5], and more recently the growth and decline of religious populations $[6,7,8,9]$. A classical mathematical method of physics are differential equations or systems of them. As an example, if the change $d n(t) / d t$ with time $t$ of a certain population $n(t)$ is proportional to $n(t)$ we immediately arrive in the simplest case at the differential equation $d n(t) / d t=C n(t)$ with $C$ as proportionality constant. Population dynamics heavily relies on such differential equations. Two prominent examples are the predator-prey dynamics and the epidemics models [10]. In this paper we will propose a differential equation system for the growth of two interacting populations where we have ideological parties or religious populations in mind among which conversion can take place.

\section{The model}

Competition among human or animal populations is a vast subject in mathematical sociology. Most studies consider food, territory or social ranks as object of the competition $[11,12]$. Here we are concerned with the competition for the number of members. Each population aims to grow in number by use of certain strategies. In principle, a certain strategy of population $\alpha$ may be neutral with respect to the growth of population $\beta$ or even beneficial ("cooperation") but most often a strategy of $\alpha$ will be harmful for $\beta$. We take into account three strategies: a) reproduction, b) conversion, and c) aggression.

In the following, populations will be designated by the index $s$ with $s=$ $1, \ldots, S$ where $S$ is the total number of populations. Then a population $s$ is represented by the number $n_{s}(t)$ of its members which depends on time $t$. Since we will consider mutually interacting populations we will need two instances of $s$ which we will designate by the indices $s a$ and $s b$ with $s a, s b=1, \ldots, S$. If we want to work with fixed values of $s a$ and $s b$ then we will use $\alpha$ and $\beta$ in order to name the two concrete populations $n_{\alpha}$ and $n_{\beta}$.

A strategy $\mathcal{S}$ is expressed as a discrete set of two values which represent the two possible realizations of $\mathcal{S}$. For $\mathcal{S}=\mathcal{I}=$ Identification we have $\mathcal{I}=$ $\left\{\{\right.$ Indolence, Emphasis $\}$. If a member $m$ of a given population $n_{s a}$ is indolent against its identity with $n_{s a}$, then $m$ is susceptible to conversion from $n_{s a}$ to

$n_{s b}$. If on the contrary $m$ is emphatic for its identity with $n_{s a}$, then it is not susceptible for conversion to $n_{s b}$. For $\mathcal{S}=\mathcal{A}=$ Activity we have $\mathcal{A}=$ \{Irenic, Aggressivity\} ${ }^{1}$. If a member $m$ of a given population $n_{s a}$ is irenic, then it will not fight for $n_{s a}$ in a conflict with another population $n_{s b}$. If on the contrary $m$ is aggressive, then it will fight for $n_{s a}$.

Now subpopulations come into play. A population $n_{s}$ is composed of

\footnotetext{
${ }^{1}$ Irenic means peaceful.
} 
subpopulations where each subpopulation represents the fraction $p_{s, v 1, v 2}$ of $n_{s}$ which carries the strategy combination (= characteristics) $\{v 1, v 2\}$ with $v 1 \in\{I n, E m\}$ and $v 2 \in\{I r, A g\}$. For example $p(s, 1,1)=p(s, I n, I r)$ is the indolent and irenic fraction of population $n_{s}$. Its contrary is $p(s, 2,2)=$ $p(s, E m, A g)$, that is the empathic and aggressive part of $n_{s}$. In total we have $2 \times 2=4$ characteristics and thus four corresponding subpopulations with $\left(p_{s, 1,1}+p_{s, 1,2}+p_{s, 2,1}+p_{s, 2,2}\right) n_{s}=n_{s}$. A subpopulation $n_{s, v 1, v 2}$ then is expressed as

$$
n_{s, v 1, v 2}=p_{s, v 1, v 2} n_{s}
$$

Populations as well as subpopulations can interact with each other. We model the interaction as the usual population product like $n_{s a} n_{s b}$. This product expresses that any member of population $n_{s a}$ can interact with any member of $n_{s b}$ which is a rough approximation of real social processes. One can think of more detailed formulations [9], in particular spatial models but here we adhere to simplicity. In terms of interacting subpopulations we then have products of the form $p_{s a, v 1 a, v 2 a} n_{s a}(t) p_{s b, v 1 b, v 2 b} n_{s b}(t)$. Then a strategy combination va1, va2 is represented by the subpopulation $p_{s a, v 1 a, v 2 a} n_{s a}(t)$. Likewise of thinking of interacting populations we can think of interacting strategies. The subpopulations are the carriers of the strategies which could not interact without such a substrate. While this notion may sound a bit esoteric, it is in fact the core of the present model.

The challenge is to formulate the interactions of strategies in a quantitative manner. The growth and decrease of interacting populations is the quantitative result of interacting strategies. In other words, the interaction of strategies is reflected in the growth and decrease of interacting populations. The use of subpopulations gives us the freedom to describe the consequences of a given strategy $\mathcal{S}$ not only among populations but also within a given population (which uses $\mathcal{S}$ ). Thus, the use of subpopulations allows us to describe sideeffects. For example, while aggression may be of advantage for population $n_{s a}$ in competition with $n_{s b}$, the aggressive subpopulation $\left(p_{s a, I n, A g}+p_{s a, E m, A g}\right) n_{s a}$ may be of disastrous effect on $n_{s a}$ itself.

We couple two strategies by the constant (not time-depending) coupling coefficient $C_{s a, s b, v a 1, v a 2, v b 1, v b 2}$ which may take on positive and negative values. Then the product

$$
\Delta=C_{s a, s b, v a 1, v a 2, v b 1, v b 2} p_{s a, v a 1, v a 2} n_{s a}(t) p_{s b, v b 1, v b 2} n_{s b}(t)
$$

is the quantitative expression of the interaction of the strategy characteristics $\{\mathcal{S}(s a, v a 1), \mathcal{S}(s a, v a 2)\}$ of population $n_{s a}$ with the strategy characteristics $\{\mathcal{S}(s b, v b 1), \mathcal{S}(s b, v b 2)\}$ of population $n_{s b}$. We will call $\Delta$ interaction term. Since we have four different characteristics, we end up with 16 combinations of interacting characteristics $\{\operatorname{In}, \operatorname{Ir}\}\{\operatorname{In}, \operatorname{Ir}\}, \ldots,\{\operatorname{In}, A g\}\{E m, A g\}$, 
$\{E m, I r\}\{I n, I r\}, \ldots,\{E m, A g\}\{E m, A g\}$. Each characteristic combination contributes to growth or decrease of the involved populations $n_{s a}$ and $n_{s b}$. We just mention that the model could be enlarged to include interactions among triples of populations etc..

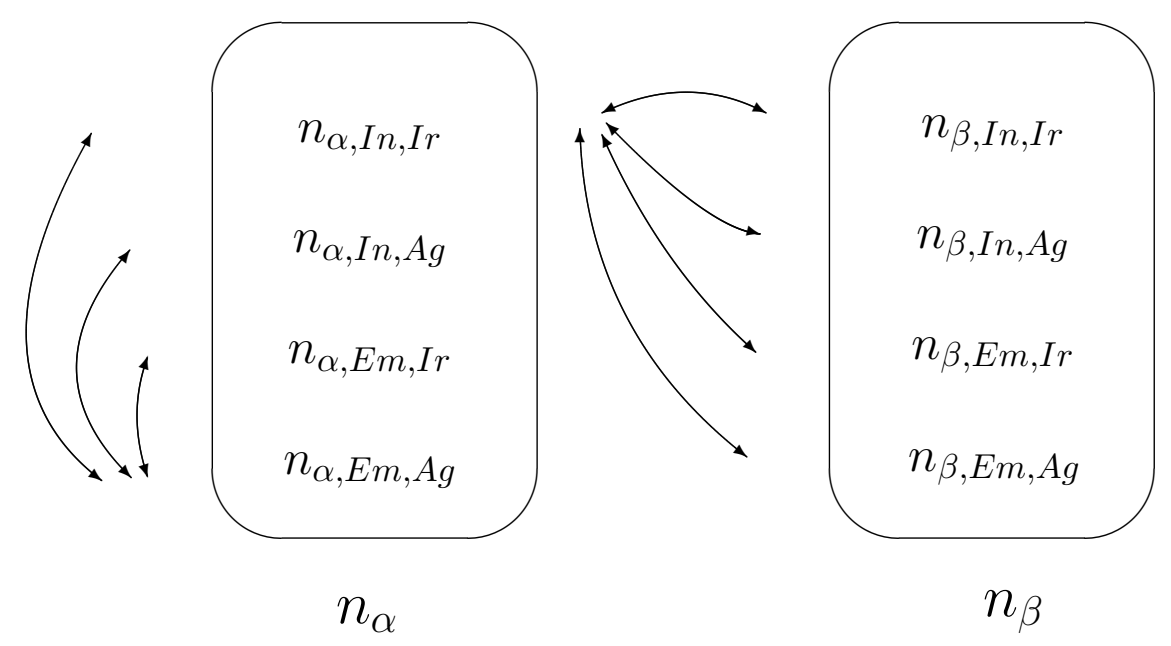

Figure 1: Intra-interactions: Each subpopulation interacts with each other supopulation within the population, e.g. subpopulation $n_{\alpha, E m, A g}(t)$ interacts with all other subpopulations of population $\alpha$. Inter-interactions: Each subpopluation interacts with each other subpopulation of another population, e.g. subpopulation $n_{\alpha, I n, I r}(t)$ interacts with all subpopulations of population $\beta$.

One final ingredient needs to be mentioned. The fraction $0 \geq p_{s, v 1, v 2} \geq$ 1 may be considered as a probability to meet a member (an "individual") $m$ of population $s \in\{s a, s b, \ldots\}$ who represents the strategy characteristics $\{v 1, v 2\}$. We now allow for a dependency of $p_{s, v 1, v 2}$ on the population numbers $n_{s a}$ and $n_{s b}$. Thus, a population may change the probability by which it follows a certain strategy. For example, if population $n_{s a}$ has been quite irenic up to now, but is threatened by extinction from aggression by population $n_{s b}$, then $n_{s a}$ may become more aggressive. In quantitative terms, we use a log-normal distribution depending on the ratio $\ln \left(n_{s a}(t) / n_{s b}(t)\right)$.

Putting all together, we arrive at the following differential equation system

$$
\begin{aligned}
\frac{d n_{s a}(t)}{d t}= & \sum_{s a=1}^{S} \sum_{s b=1}^{S} \sum_{\substack{v a 1 \in \\
\{\mathrm{In}, \mathrm{Em}\}}} \sum_{\substack{v a 2 \in \\
\{\mathrm{r}, \mathrm{Ag}\}}} \sum_{\substack{v b 2 \in \\
\{\mathrm{In}, \mathrm{Em}\}}} \sum_{\substack{v b 2 \in \\
\{\mathrm{r}, \mathrm{Ag}\}}} \\
& C_{s a, s b, v a 1, v a 2, v b 1, v b 2} p_{s a, v a 1, v a 2}\left(n_{s a}, n_{s b}\right) n_{s a}(t) p_{s b, v b 1, v b 2}\left(n_{s a}, n_{s b}\right) n_{s b}(t)
\end{aligned}
$$

where $s a, s b=1, \ldots, S$ and $S=2$ in the present study. 
Care must be taken concerning the balancing of the different contributions $\Delta=C_{s a, s b, v a 1, v a 2, v b 1, v b 2} p_{s a, v a 1, v a 2} n_{s a}(t) p_{s b, v b 1, v b 2} n_{s b}(t)$ to (3). A certain $\Delta$ may remove members from $n_{s a}(t)$ at time $t$ which corresponds to "death" in our model. Another $\Delta^{*}$ may embody the conversion process from $n_{s a}$ to $n_{s b}$, then the loss for $\alpha$ is $d n_{s a} / d t=-\Delta^{*} \pm \ldots$ and the corresponding gain for $\beta$ is $d n_{s b} / d t=\Delta^{*} \pm \ldots$

\section{$3 \quad \beta$ growing a the cost of $\alpha$ and a remedy}

Consider a population $\beta$ of size $n_{\beta}(t)$ which is growing at the cost of population $\alpha$ of size $n_{\alpha}(t)$. Such a growth can be achieved by conversion of members of $\alpha$ to $\beta$. We assume that aggressive members of $\beta$ with emphasis on their identity cause the conversion. This subpopulation is designated as $p_{\beta, E m, A g} n_{\beta}$. Indolent members of $\alpha$ who do not exhibit a strong identification with $\alpha$ are most susceptible to conversion. These indolent members form the subpopulation $\left(p_{\alpha, I n, I r}+p_{\alpha, I n, A g}\right) n_{\alpha}$ and we express their susceptibility to conversion to $\beta$ by coefficients $C_{\alpha, \beta, I n, A g, E m, A g}$ and $C_{\alpha, \beta, I n, A g, E m, A g}$ which both assume a negative value (of -0.0005 for the present example).

At this point we discuss the amounts of the coefficients $C_{s a, s b, v a 1, v a 2, v b 1, v b 2}$. The dimension of $C_{s a, s b, v a 1, v a 2, v b 1, v b 2}$ is $1 / \mathrm{T} / \mathrm{P}^{2}$ ) where $\mathrm{T}$ stands for the unit of time and $\mathrm{P}$ stands for "person". Thus $C_{s a, s b, v a 1, v a 2, v b 1, v b 2}=f$ is the frequency which determines the number of interactions per time unit among the subpopulations $n_{s a}$ and $n_{s b}$. In a model calculation we will choose as observation time a multiple $x T$ of the period $T=1 / f$ such that $x T>n_{s a} n_{s b}$. The challenging question is: How great are these frequencies? Obviously, the answer is difficult to give due to the complexity of real social processes and any answer will be a simplification. For the present study, we just assume that $C_{s a, s a, v a 1, v a 2, v b 1, v b 2}$ is in the order of $\approx 0.001$ for intra-population interaction processes and $C_{s a, s b, v a 1, v a 2, v b 1, v b 2}$ is in the order of $\approx 0.0001$ for inter-population interaction processes.

If a member $a \in \alpha$ puts emphasis on his membership to population $\alpha$, then he is more resistant to conversion from $\alpha$ to $\beta$ than a different member $a^{*} \in \alpha$ which is indolent with respect to (insensible to) his membership. For our present example we assume that both $\alpha$ and $\beta$ have the same fraction $=$ $20 \%$ of indolent members and thus a fraction $=80 \%$ of empathic members. If a member $a \in \alpha$ is aggressive, then he is more probable to lead a member $b \in \beta$ to conversion from $\beta$ to $\alpha$ than an irenic member $a^{*} \in \alpha$. For our present example we assume that $\alpha$ has $30 \%$ aggressive members but $\beta$ is a much more aggressive population with $60 \%$ aggressive members. Then we can calculate the combined fractions $p_{\alpha, v 1 a, v 2 a}$ and $p_{\beta, v 1 b, v 2 b}$ as corresponding products, e.g. $p_{\alpha, I n, I r}=0.2 \times(1-0.3)=0.14$ or $p_{\beta, E m, A g}=0.8 \times 0.6=0.48$.

Reproduction is the most important strategy for any population. We 
have to set the corresponding coupling coefficients $C_{\alpha, \alpha, v a 1, v a 2, v b 1, v b 2}$ for $n_{\alpha}$ and $C_{\beta, \beta, v a 1, v a 2, v b 1, v b 2}$ for $n_{\beta}$ with $v a 1 \in\{I n, E m\}$ and $v a 2 \in\{I r, A g\}$. Assuming that there are no preferences or preclusions among subpopulations we put $C_{\alpha, \alpha, v a 1, v a 2, v b 1, v b 2}=0.001$ and $C_{\beta, \beta, v a 1, v a 2, v b 1, v b 2}=0.0012$ which means that $n_{\beta}$ is more fertile than $n_{\alpha}$. However, here we keep the populations strictly separated with respect to reproduction, thus $C_{\alpha, \beta, v a 1, v a 2, v b 1, v b 2}=C_{\beta, \alpha, v a 1, v a 2, v b 1, v b 2}=0$. At time $t=0$ we have to provide stating values $n_{\alpha}(t=0)$ and $n_{\beta}(t=0)$. For the present example we put $n_{\alpha}(t=0)=n_{\beta}(t=0)=100$ members.

Furthermore the values of the coupling coefficients $C_{s a, s b, v a 1, v a 2, v b 1, v b 2}$ need to be fixed. We read the dependency on $\alpha, \beta$ as action of $\beta$ onto $\alpha$. The action (interaction) is expressed in terms of $d n_{\alpha} / d t$ and $d n_{\beta} / d t$ in the frame of eq. (3). For example, for a conversion from the indolent and irenic subpopulation $p_{\alpha, I n, I r} n_{\alpha}$ of $\alpha$ to $\beta$ by interaction with the emphatic and aggressive subpopulation $p_{\beta, E m, A g} n_{\beta}$ of $\beta$ we observe a fraction $C_{\alpha, \beta, I n, I r, E m, A g} p_{\alpha, I n, I r} n_{\alpha} p_{\beta, E m, A g} n_{\beta}$ of converted members. In the present example we take $C_{\alpha, v a 1, v a 2, E m, A g}=0.0005$ for the combinations $\{v a 1=I n, v a 2=I r\},\{v a 1=I n, v a 2=A g\}$, $\{v a 1=E m, v a 2=I r\}$ but $C_{\alpha, v a 1, v a 2, E m, A g}=0.0$ for $\{v a 1=E m, v a 2=A g\}$. So $\alpha$ is quite susceptible to conversion by aggression exerted by $\beta$. On the other hand we assume that $n_{\beta}$ does not suffer any conversion of its members to $n_{\alpha}$.

Many more interactions could be modeled, in particular deadly fights among $\alpha$ and $\beta$ (i.e. inter-population) as well among subpopulations of a given population (i.e. intra-population). Concerning inter-population aggression we assume for the present example that the losses for $n_{\beta}$ are higher than for $n_{\alpha}$ with $C_{\alpha, \beta, v a 1, v a 2, v b 1, v b 2}=-0.00001$ and $C_{\beta, \alpha, v a 1, v a 2, v b 1, v b 2}=-0.00005$. Losses due to intra-population aggression is more probable the more aggressive a population is. This is a case of a disadvantageous side-effect of a strategy: Aggressivity may be of advantage in the inter-population interaction but may lead to inter-population fights, too.

We are ready to carry out a first evaluation of the equation system (3). Inserting the discussed fractions of subpopulations and the selected coupling coefficients we finally arrive at a rather simple differential equation system (called case 1$)^{2}$.

$$
\begin{aligned}
& \frac{d n_{\alpha}(t)}{d t}=0.00050 n_{\alpha}(t)^{2}-0.00019 n_{\alpha}(t) n_{\beta}(t) \\
& \frac{d n_{\beta}(t)}{d t}=0.00018 n_{\alpha}(t) n_{\beta}(t)+0.00050 n_{\beta}(t)^{2}
\end{aligned}
$$

Here we are interested in the time-dependent developments of $n_{\alpha}(t)$ and $n_{\beta}(t)$. Thus, a numerical solution of eq. (3) will be sufficient and we arrive at

\footnotetext{
2 "The mountains will be in labor, and a ridiculous mouse will be brought forth.", Quintus Horatius Flaccus $(65-8 \mathrm{BC})$.
} 
fig. 2. Obviously, population $\alpha$ is threatened with extinction. The indolent and irenic subpopulations are too much accessible to conversion.

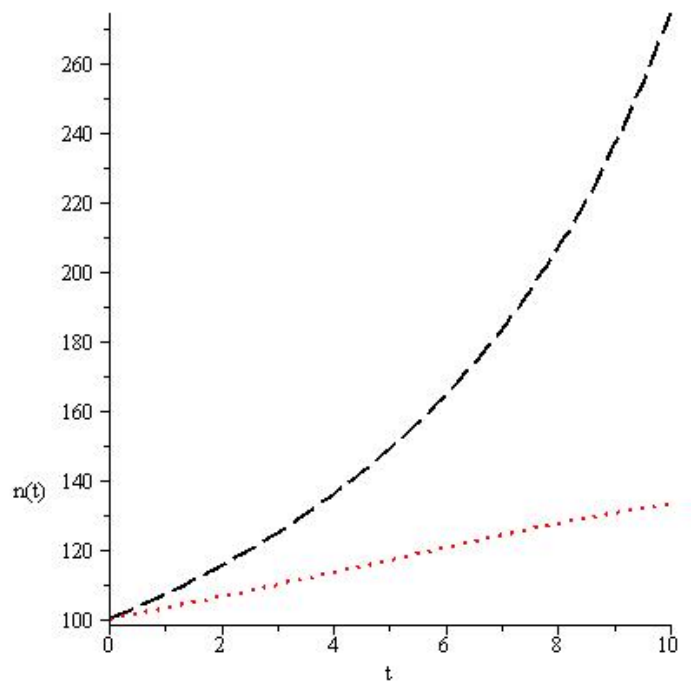

Figure 2: Solution to (4). Dotted line $=n_{\alpha}(t)$, dashed line $n_{\beta}(t)$.

Several mechanisms can be imagined to improve the growth of $\alpha$. One can think of a change $\delta C$ in the amounts of the coupling coefficients. Many process of a real-world situation could be linked to $\delta C$. For example education could lead to stronger identification. Another route consists in the change of the fractions of the subpopulations. Here we take this route. The irenic and aggressive fractions become dependent on the ratio $n_{\alpha}(t) / n_{\beta}(t)$ according to a log-normal (distribution) function

$$
p_{\alpha, I r}=1.0-\exp \left(-\frac{\left(\ln \left(\frac{n_{\alpha}(t)}{n_{\beta}(t)}\right)-\ln (1.0)\right)^{2}}{2 \sigma_{\alpha}^{2}}\right)
$$

where $\sigma_{\alpha}$ is a parameter. With (6) the irenic fraction will get smaller the smaller the ratio $n_{\alpha}(t) / n_{\beta}(t)$ will get. Since $p_{\alpha, I r}+p_{\alpha, A g}=1$ the aggressive fraction of $\alpha$ will grow in less favorable situations for $\alpha$ and thus the subpopulation $p_{\alpha, E m, A g}$ will grow which is not susceptible to conversion. A simliar mechanism is implemented for $\beta$. With $\sigma_{\alpha}=\sigma_{\beta}=0.5$ we arrive at a more voluminous differential equation system which is too long to be displayed here but called "case 2" in the following.

The numerical solution of case 2 is displayed in fig. 3. Obviously, population $\alpha$ will survive in the second case. The increase of $p_{\alpha, E m, A g}\left(n_{\alpha}(t), n_{\beta}(t)\right)$ with decreasing $n_{\alpha}(t) / n_{\beta}(t)$ produces a subpopulation $p_{\alpha, E m, A g}\left(n_{\alpha}(t), n_{\beta}(t)\right) n_{\alpha}(t)$ which compensates the loses due to conversion. 


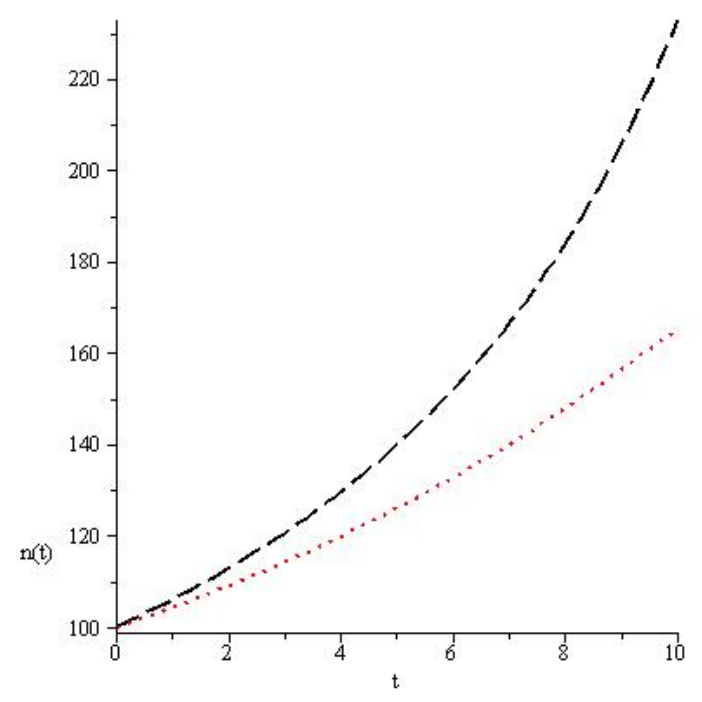

Figure 3: Solution to case 2. Dotted line $=n_{\alpha}(t)$, dashed line $n_{\beta}(t)$.

\section{Discussion}

\subsection{Dynamcis}

The differential equation system (3) can be understood as a dynamical system. Any usual discussion of a such a dynamical system looks at its stability points where $d n_{s}(t) / d t=0$ for all populations $s=1, \ldots, S$. Then we want to find stability points other that the trivial point $n_{s}=0$. Thus, in the classical approach one will consider $n_{s a}$ and $n_{s b}$ as the independent variables in order to solve (7).

$$
\begin{aligned}
0= & \sum_{s a=1}^{S} \sum_{s b=1}^{S} \sum_{\substack{v a 1 \in \\
\{\mathrm{In}, \mathrm{Em}\}}} \sum_{\substack{v a 2 \in \\
\{\mathrm{Ir}, \mathrm{Ag}\}}} \sum_{\substack{v b 2 \in \\
\{\mathrm{In}, \mathrm{Em}\}}} \sum_{\substack{v b 2 \in \\
\{\mathrm{Ir}, \mathrm{Ag}\}}} \\
& C_{s a, s b, v a 1, v a 2, v b 1, v b 2} p_{s a, v a 1, v a 2}\left(n_{s a}, n_{s b}\right) n_{s a}(t) p_{s b, v b 1, v b 2}\left(n_{s a}, n_{s b}\right) n_{s b}(t)
\end{aligned}
$$

For any reasonable discussion of stability points the system should be equipped with creation and discreation process which can equilibrate each other. Otherwise the system will not have any stability points. In the above formulation of (3) we did not include a non-violent decease process which could compensate the reproduction process which has been included. Thus, in any broader discussion of (3) we implicitly assume a sufficient enrichment of (3) with suitable interaction processes.

However, we are not interested in $n_{s}$ as the independent variables. The dynamical system in mind is not so much composed of interacting populations 
$n_{s}$, but composed of interacting strategies weighted by subpopulations which carry the strategies. Consequently, we are interested in $p_{s, v 1, v 2} n_{s}(t)$ as the independent variables (where we dropped in the first stage the dependency of $p$ on $\left.n_{s a}, n_{s b}\right)$. The main question is: Which strategy combination should population $n_{s}$ choose by appropriate magnitudes of its subpopulations $p_{s, v 1, v 2} n_{s}(t)$ in order to survive at a constant population number $d n_{s} / d t=0$ or even better at a growth rate $d n_{s} / d t>0$ ?

Obviously the answer depends on the interactions and their coupling constants given in the dynamical system (7). At that stage we can introduce the notion of the "scenario": The set of coupling constants $C_{s a, s b, v a 1, v a 2, v b 1, v b 2}$ with $s a, s b=1, \ldots, S$ and $v a 1, v b 1 \in\{I n, E m\}, v a 2, v b 2 \in\{\operatorname{Ir}, A g\}$ is the "scenario" in our model world. A population $n_{s a}$ is partitioned into subpopulations and the partition will result in a certain value for $d n_{s a} / d t$ depending on the given scenario $C_{s a, s b, v a 1, v a 2, v b 1, v b 2}$. Aggressive subpopulations may serve as illustration for side effects within the scenario: While aggressivity may be of advantage in the interaction with other populations $n_{s b}$, agressivity may be harmful in the internal interaction within the population $n_{s a}$ (for this case $s b=s a)$. Since aggressivity may lead to a death toll within $n_{s a}$ according to $C_{s a, s a, v a 1, v a 2, v b 1, A g} p_{s a, v a 1, v a 2} n_{s a} p_{s a, v b 1, A g} n_{s a}$ with va1,va2 $\in\{I n, E m, \operatorname{Ir}, A g\}$, $v b 1 \in\{I n, E m\}$.

With a given scenario we can solve (7) for a certain subpopulation, e.g. for $p_{\alpha, E m, A g} n_{\alpha}$ which has played a prominent role in our above example. Let $(1-\delta(s a=\alpha, s b=\beta, v a 1=E m, v a 2=A g)))=0$ if we observe $s a=\alpha, s b=$ $\beta, v a 1=E m, v a 2=A g$ simultaneously (i.e. $\delta$ is a Kronecker delta). From the analytical form of the solution (8) with its many summands (depending on the model complexity) we learn that any interpretation will be difficult.

$$
\begin{array}{r}
\sum_{\substack{v b 1 \in \\
\{\mathrm{In}, \mathrm{Em}\}\}}} \sum_{\substack{v b 2 \in \\
\{\mathrm{Ir}, \mathrm{Ag}\}}} C_{\alpha, \beta, \mathrm{Em}, \mathrm{Ag}, v b 1, v b 2} p_{\alpha, \mathrm{Em}, \mathrm{Ag}} n_{\alpha}(t) p_{\beta, v b 1, v b 2} n_{\beta}(t)= \\
\sum_{s a=1}^{S} \sum_{s b=1}^{S} \sum_{\substack{v a 1 \in \\
\{\mathrm{In}, \mathrm{Em}\}}} \sum_{\substack{v \mathrm{a} 2 \in \\
\{\mathrm{Ir}, \mathrm{Ag}\}}} \sum_{\substack{v b 1 \in \\
\{\mathrm{In}, \mathrm{Em}\}}} \sum_{\substack{v b 2 \in \\
\{\mathrm{r}, \mathrm{Ag}\}}} \\
(1-\delta(s a=\alpha, s b=\beta, v a 1=E m, v a 2=A g)) \\
C_{s a, s b, v a 1, v a 2, v b 1, v b 2} p_{s a, v a 1, v a 2} n_{s a}(t) p_{s b, v b 1, v b 2} n_{s b}(t)
\end{array}
$$

The next question is: How does a population $n_{\text {sa }}$ find a strategy combination which results in $d n_{s a} / d t>0$ ? In this connection we just want to point out an alternative view on (7). We can concentrate on the sets of the coupling coefficients and ask which combinations of their values is most favourable (or most harmful) for a stable population growth ${ }^{3}$. Thus, we arrived at an optimisation

\footnotetext{
${ }^{3}$ According to the philosopher Leibniz our world is the best possible world since it was
} 
problem with the coupling coefficients $C_{s a, s b, v a 1, v a 2, v b 1, v b 2}$ as the independent variables. However, the scenario has a certain structure, that is, certain relationships among the coupling coefficients. These relationships extend from fundamentals like physical conservation laws to economical boundary conditions. In addition, the coupling coefficients may depend on time! In fact, the determination of these relationships and boundary conditions is a complex task for itself and we do not pursue this task further here.

At this point we want to focus the discussion on the strategies. A strategy $v a 1, v a 2$ is represented by the subpopulation $p_{s a, v 1 a, v 2 a} n_{s a}(t)$ and the success for $n_{s a}$ in interaction with $n_{s b}$ (competition or cooperation) depends on the interaction term $\Delta=C_{s a, s b, v a 1, v a 2, v b 1, v b 2} p_{s a, v a 1, v a 2} n_{s a}(t) p_{s b, v b 1, v b 2} n_{s b}(t)$. We are well aware that our quantitative description of strategies is a strong simplification. Perhaps the most important shortcoming in our model is that no awareness is attached to their application. In our model no self-aware person does apply a certain strategy. A person could ponder the strategies and it could choose among them. Furthermore, a person would be able to further develop strategies. On the other hand, all these process are extremely difficult to model. In the frame of our model we purposely map these processes onto unaware subpopulations.

Concerning strategies a final main question should be addressed: Where do the strategies emerge from? In our understanding this question ranges from science to philosophy. A strategy is a behavior in order to attain a certain aim. Where does such a behavior come from? The ansatz of evolutionary models in biology is to explain the origin and development of species from a interaction among them where species actually explore different strategies by trail and error. Our model is quite close to this ansatz. However, the question remains, where do the strategies emerge from? The notion of emergence which means the coming into being of something by interaction of constitutional parts. It seems to be quite difficult to reduce a strategy into constitutional parts and to transfer the origin question onto these parts. Such an approach may also be termed a reductionistical explanation. In our model, we have to introduce any strategy as a whole and ad hoc. If we introduce model elements ad hoc, then we are in danger to formulate our model arbitrarily.

\subsection{From the sociological point of view}

The sociological reality is overwhelmingly complex and thus we have to make certain simplifications if we try to build a sociophysical model. In the present approach we established the strategies on the level of subpopulations. This approach allows us the use of differential equations in order to describe the growth and decline of populations. However, maybe strategies should be lo-

created by a good and almighty God (Leibniz, 1646 - 1716). 
cated at the level of individuals. Then we have to work with sets of individuals which makes the mathematical description far more complicated. A popular method on the individual level are the agent-based models [1, 14]. In fact, this interplay of individual behavior based on certain strategies and the overall population behavior is at the heart of sociological interest and our model is just a simplifying view on it.

From the sociological point of view our model may appear ridiculously simple. A sociologist may accuse us of dumbing down sociological processes to simple arithmetic relations. Where is our justification? A quantitative model of a certain subject is scientifically justified if it provides assertions about its subject which can be proven from comparison with measurable quantities of the subject. However, in the present paper we did not intend to provide testable assertions but to introduce a model which possibly can be developed into a state where it produces testable predictions on population growth. At its present stage, the model returns reasonable results within the frame of its premises and assumptions.

The wealth of sociological phenomena can not be mapped fully onto a simple differential equation system. One may take for example our term "Identification" as one of the basic strategies. Actually, identification is a most important sociological strategy. A hierarchy with a representative person at the top ("king") give its members the opportunity to identify with the hierarchy via identification with the "king". Hence a king will have to be visible for the hierarchy members and will have to provide suitable signs. This circumstance give rise to many sociological phenomena. Undeniably we have a gap between our meager differential equation system (3) and the social reality.

\section{Conclusion}

A stable society relies on the balance of its strategies. Eq. (7) is a first rough formalization of this idea. Then an unbalance will cause a destabilization. The unbalance may arise from an exaggerated use of a certain strategy. In a certain sense, the strategy takes on a life of its own. We have in mind the exaggerated use of social identity. Social catastrophes like confessional wars may be seen under this aspect. Further studies may throw more light on the interplay of strategies.

\section{Appendix}

A maple program for setting up the equation system (3) and for its solution is available at: https://googledrive.com/host/0BwBiT53KUxq-ZDhOdGQ4M0hBTWc/species3.mpl 
For an introduction into scientific modeling using Maple see [13].

\section{References}

[1] C. Castellano, S. Fortunato, V. Loreto: Statistical physics of social dynamics, Reviews of Modern Physics, 81(2), (2009), 591-646, http://dx.doi.org/10.1103/revmodphys.81.591.

[2] Serge Galam: Sociophysics, Springer Verlag, Heidelberg, 2012, ISBN 9781-4614-2032-3, http://dx.doi.org/10.1007/978-1-4614-2032-3.

[3] Dietrich Stauffer: Sociophysics simulations II: opinion dynamics, edited by: Joaquin Marro, Pedro L. Garrido, Miguel A. Munoz MODELING COOPERATIVE BEHAVIOR IN THE SOCIAL SCIENCES, 779(1), (2005), 56-68, arXiv:physics/0503115v1 [physics.soc-ph] 14 Mar 2005.

[4] Nikolay K. Vitanov, Zlatinka I. Dimitrova, Marcel Ausloos: A model of ideological struggle, Physica A 389 (2010) 4970-4980, http://arxiv.org/abs/0906.4962.

[5] Arvind Kumar Misra: A simple mathematical model for the spread of two political parties, Nonlinear Analysis: Modelling and Control 17(3) (2012), 343-354.

[6] M. Ausloos: On religion and language evolutions seen through mathematical and agent based models, Proceedings of the First Interdisciplinary Chess Interactions Conference (World Scientific, Singapore, 2010) pp. 157182, http://arxiv.org/abs/1103.5382.

[7] D. M. Abrams H. A. Yaple, and R. J. Wiener, Dynamics of social group competition: modeling the decline of religious affiliation, Physical Review Letters 107 (2011), http://dx.doi.org/10.1103/PhysRevLett.107.088701.

[8] Thomas Wieder: A simple differential equation system for the description of competition among religions, International Mathematical Forum 6(8) (2011), 1713 - 1723, http://m-hikari.com/imf-2011/33-362011/wiederIMF33-36-2011.pdf.

[9] Thomas Wieder: Addendum to "A simple differential equation system for the description of competition among religions"; International Mathematical Forum 7(54) (2012), 2681 - 2686, http://m-hikari.com/.

[10] D.S. Jones and B.D Sleeman: Differential Equations and Mathematical Biology, George Allen \& Unwin Ldt., London, 1983, ISBN 0-04-515001-X 
[11] E. Ben-Naim, F. Vazquez, S. Redner: On The Structure of Competitive Societies, The European Physical Journal B 49, 531-538 (2006).

[12] K. Kondala Rao and K. Lakshmi Narayan: Stability analysis of a three species syn-eco dynamical system with a limited alterantive food for all three species, Bulletin of Society for mathematical services \& standards 1(1) (2012), 38-48.

[13] Richard H. Enns and George C. McGuire: Computer Algebra Recipes An Advanced Guide to Scientific Modeling, Springer-Verlag, Heidelberg, 2007, ISBN-13: 978-387-25768-6.

[14] Paul Smaldino, Cynthia Pickett, Jeffrey Sherman and Jeffrey Schank (2012): An Agent-Based Model of Social Identity Dynamics, Journal of Artificial Societies and Social Simulation 15(4) 7, http://jasss.soc.surrey.ac.uk/15/4/7.html.

\section{Received: October 1, 2013}

